\title{
Measurement of intracellular strain on deformable substrates with texture correlation
}

\author{
Christopher L. Gilchrist ${ }^{\mathrm{a}}$, Sietske W. Witvoet-Braam ${ }^{\mathrm{b}}$, Farshid Guilak ${ }^{\mathrm{c}}$, Lori A. Setton ${ }^{\mathrm{a}, \mathrm{c}, *}$ \\ ${ }^{a}$ Department of Biomedical Engineering and Surgery, Duke University, 136 Hudson Hall, Box 90281, Durham, NC 27708, USA \\ ${ }^{\mathrm{b}}$ Department of Biomedical Engineering, Eindhoven University of Technology, Eindhoven, The Netherlands \\ ${ }^{\mathrm{c}}$ Division of Orthopaedic Surgery, Department of Surgery, Duke University Medical Center, Durham, NC, USA
}

Accepted 24 March 2006

\begin{abstract}
Mechanical stimuli are important factors that regulate cell proliferation, survival, metabolism and motility in a variety of cell types. The relationship between mechanical deformation of the extracellular matrix and intracellular deformation of cellular subregions and organelles has not been fully elucidated, but may provide new insight into the mechanisms involved in transducing mechanical stimuli to biological responses. In this study, a novel fluorescence microscopy and image analysis method was applied to examine the hypothesis that mechanical strains are fully transferred from a planar, deformable substrate to cytoplasmic and intranuclear regions within attached cells. Intracellular strains were measured in cells derived from the anulus fibrosus of the intervertebral disc when attached to an elastic silicone membrane that was subjected to tensile stretch. Measurements indicated cytoplasmic strains were similar to those of the underlying substrate, with a strain transfer ratio (STR) of 0.79 . In contrast, nuclear strains were much smaller than those of the substrate, with an STR of 0.17. These findings are consistent with previous studies indicating nuclear stiffness is significantly greater than cytoplasmic stiffness, as measured using other methods. This study provides a novel method for the study of cellular mechanics, including a new technique for measuring intranuclear deformations, with evidence of differential magnitudes and patterns of strain transferred from the substrate to cell cytoplasm and nucleus.
\end{abstract}

(C) 2006 Elsevier Ltd. All rights reserved.

Keywords: Cell; Mechanics; Nucleus; Strain; Extracellular matrix; Intervertebral disc

\section{Introduction}

Cells are exposed to a variety of mechanical stimuli under normal physiological conditions including deformations, hydrostatic pressures and fluid flow. Cells interpret these events, in combination with biochemical and bioelectrical stimuli, as signals that influence or control cell proliferation, survival, differentiation, metabolism and motility (Guilak et al., 1997; Estes et al., 2004; Hsieh and Nguyen, 2005; Li et al., 2005). The

\footnotetext{
${ }^{*}$ Corresponding author. Department of Biomedical Engineering and Surgery, Duke University, 136 Hudson Hall, Box 90281, Durham, NC 27708, USA. Tel.: + 19196605131 ; fax: + 19196818490 .

E-mail address: setton@duke.edu (L.A. Setton).
}

means by which cells sense mechanical stimuli and translate them into intracellular biochemical responses (i.e. "mechanotransduction") are the subject of much study and are not fully understood (Iqbal and Zaidi, 2005). At the cell surface, transmembrane proteins including integrins (Katsumi et al., 2004) and mechanosensitive ion channels (Sachs and Morris, 1998) have been implicated in the transduction of mechanical stimuli. Mechanical signals may be transmitted across the cell membrane to focal adhesion complexes and the cytoskeleton (Bershadsky et al., 2003; Shemesh et al., 2005), or cytoskeletal components may provide a direct mechanical link to transmit signals from the extracellular matrix to the cell nucleus (Maniotis et al., 1997; Chen and Ingber, 1999). Together these components 
may transfer mechanical stimuli to particular locations within the cell, with spatial localization of the signal potentially yielding a specific intracellular response $(\mathrm{Hu}$ et al., 2005; Wang and Suo, 2005).

A number of techniques have been developed for applying mechanical stimulation to cultured cells (reviewed in (Brown, 2000)), including methods that apply controlled bending, uniaxial strain or multiaxial strain to deformable substrates (Banes et al., 1985; Hung and Williams, 1994; Lee et al., 1996; Bottlang et al., 1997). Although various theoretical and experimental studies have been performed to determine the complex strain profiles that may exist at the substrate surface in these devices (Gilbert et al., 1994; Brown et al., 2000; Jahangir et al., 2002; Vande Geest et al., 2004), little is known regarding how the imposed substrate deformations are related to deformations generated within the sub-regions of an attached cell.

Several approaches have been utilized to study intracellular deformation in response to mechanical stimulation. For example, ligand-coated magnetic beads have been used to apply mechanical forces or displacements at discrete locations on single cells and measurements of intracellular displacements made via light or fluorescence microscopy (Maniotis et al., 1997; Hu et al., 2003). Intermediate filament deformation has also been tracked in endothelial cells subjected to fluid flow using a GFP-label (Helmke et al., 2003). In one study, fluorescent latex beads were injected into a cell to measure cytoplasmic deformations, and a fluorescent nuclear dye used to measure change in nuclear dimensions following tensile stretch of a deformable substrate (Caille et al., 1998). This method allowed estimation of the stretch transferred from the substrate to cytoplasmic and nuclear regions of the cell, although estimates of strain were only possible over large areas of the cytoplasm $\left(\sim 25-100 \mu \mathrm{m}^{2}\right)$ and the entire nucleus $\left(\sim 100 \mu \mathrm{m}^{2}\right)$.

In this study, we present a novel approach to measure high-resolution intracellular and intranuclear strains in cells attached to a deformable substrate using confocal microscopy and an image analysis method, texture correlation. The primary objective of this study was to examine the hypothesis that tensile stretch of a substrate is transferred fully (i.e. magnitude of cell strains not different from underlying substrate strains) to cytoplasmic and nuclear regions of attached cells over a range of applied substrate stretch. Fibroblast-like cells were isolated from the fibrous anulus region of intervertebral disc tissues and labeled with fluorescent probes specific to mitochondria or nuclei for visualization with confocal microscopy and tracking using two-dimensional (2D) texture correlation (Gilchrist et al., 2004). A secondary objective was to evaluate the dependence of strain transfer on cell alignment relative to the direction of substrate stretch.

\section{Materials and methods}

\subsection{Primary cell isolation and culture}

Cells were isolated from anulus fibrosus regions of freshly harvested porcine intervertebral discs $(\sim \operatorname{six}$ discs each from $N=5$ spines, $\sim 9$ weeks old) via enzymatic digestion (Baer et al., 2001). Fibrous anulus tissue was visually identified and dissected, with care taken to remove any cartilagenous tissue of the innermost anulus or nucleus regions. Cells from the anulus fibrosus exhibit a fibroblast-like phenotype, as indicated by their elongated cell morphology in vitro and in vivo, and synthesis of type I collagen (Wang et al., 2001; Horner et al., 2002). Cells were cultured $\left(37^{\circ} \mathrm{C}, 5 \% \mathrm{CO}_{2}\right)$ in subconfluent monolayers for 4-10 days in F-12 medium with $10 \%$ FBS, $10 \mathrm{mM}$ HEPES and antibiotics.

Silicone elastic membranes (SILTEC gloss membrane, $35 \mathrm{~mm} \times 10 \mathrm{~mm} \times 0.25 \mathrm{~mm}$ thickness, Technical Products, Inc., Decatur, GA) were coated on one side with a thin layer of fluorescent microspheres (Fluospheres, Ex/Em: $535 / 575 \mathrm{~nm}, 2 \mu \mathrm{m}$ diameter, Molecular Probes, Eugene, OR) suspended in silicone adhesive (GE RTV 108, Wilton, CT) to provide markers for tracking substrate deformation and sterilized under UV light for $30 \mathrm{~min}$. The opposite side of the membrane was coated with type I bovine collagen (Sigma, $40 \mu \mathrm{g} / \mathrm{mL}$ in PBS, overnight at $4{ }^{\circ} \mathrm{C}$ ) to promote cell attachment. Coated membranes were rinsed in PBS, placed into culture dishes and seeded with 10,000 cells $/ \mathrm{cm}^{2}$. Cells were cultured in the same conditions described above for $48 \mathrm{~h}$ to allow for attachment.

Prior to the stretch experiments, cell nuclei and mitochondria were fluorescently labeled using SYTO $82(10 \mu \mathrm{M}$, Ex/Em: 541/560 nm, Molecular Probes) and MitoTracker Deep Red 633 (1.5 $\mu \mathrm{M}$, Ex/Em: 644/ $665 \mathrm{~nm}$, Molecular Probes), respectively.

\subsection{Stretch experiments}

A micrometer-controlled displacement device was used to apply uniaxial stretch to the membrane (Fig. 1, modified from (Guilak et al., 1995)). The membrane was attached to the platens with the cell attachment surface inverted. The membrane was submerged in a chamber filled with warmed media $\left(37^{\circ} \mathrm{C}\right)$, which was mounted on the stage of an inverted confocal laser scanning microscope (Zeiss LSM 510, Carl Zeiss Inc., Thornwood, NY). The attached cells (inferior surface) and membrane markers (superior surface) were visualized through a coverslip window in the bottom of the chamber.

Prior to application of stretch, initial reference images of cell mitochondria and nuclei were recorded $(63 \times$ water immersion objective, NA $1.2,0.7 \times$ zoom, $206 \mu \mathrm{m} \times 206 \mu \mathrm{m}$ image field, $0.2 \mu \mathrm{m} /$ pixel resolution, 
$2.0 \mu \mathrm{m}$ slice thickness for mitochondria, $4.0 \mu \mathrm{m}$ thickness for nuclei) (Fig. 2). After imaging, the focus was adjusted to visualize the fluorescent microspheres on the superior surface of the membrane $(20 \times$ objective, NA $0.5,460 \mu \mathrm{m} \times 460 \mu \mathrm{m}$ image field, $0.45 \mu \mathrm{m} /$ pixel) (Fig. 2). A uniaxial ( $x$-direction) stretch was then applied to the membrane via controlled displacement of the micrometer at a strain rate of approximately $0.002 \mathrm{~s}^{-1}$ to achieve substrate strains between 0.05-0.15. After application of membrane stretch, digital images were immediately acquired of the deformed substrate and cells.

\subsection{Displacement measurement and strain calculations}

Strains were measured using a 2D texture correlation algorithm described previously (Gilchrist et al., 2004). Briefly, texture correlation is a subset of digital image correlation where pixel displacements are determined by comparing intensity patterns between two images, a "reference" and "deformed" image set (Bay, 1995; Bey et al., 2002; Wang et al., 2003). A pixel within the reference image is identified by a square subset of surrounding pixels (subset mask, $m \times m$ pixels) and its position in the deformed image determined via a

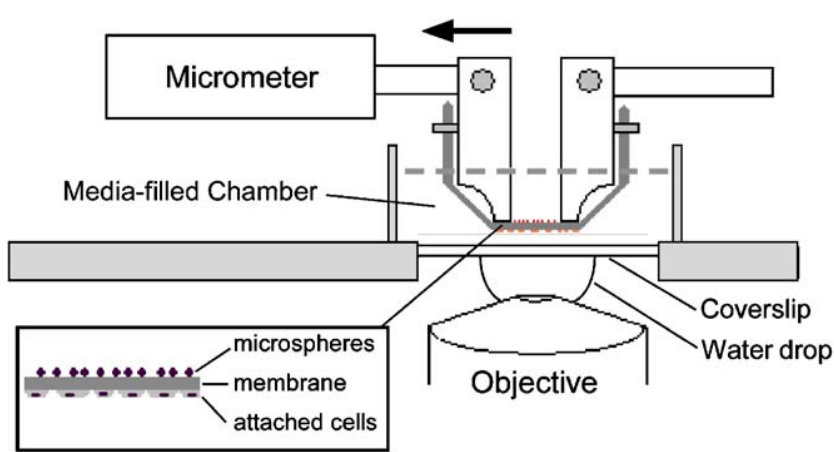

Fig. 1. Schematic of micrometer device used to apply uniaxial stretch to cell-seeded membranes. correlation algorithm. The algorithm uses a modified Newton-Raphson search procedure to maximize a correlation coefficient for each pixel, identifying the pixel's displaced position in the deformed image with sub-pixel accuracy. The algorithm incorporates firstorder displacement mapping terms to account for distortion of the subset mask in the deformed configuration, terms that were shown previously to reduce errors associated with strain calculation (Gilchrist et al., 2004). Continuous displacement fields for a grid of tracked pixels are then calculated using a bicubic smoothing spline (MATLAB function csaps.m, The Mathworks, Inc.) and differentiated to determine the 2D components of Lagrangian finite strain $\left(E_{x x}, E_{y y}, E_{x y}\right)$.

\subsection{Error analyses}

Analyses were performed to estimate error in strain measurement due to the imaging system and/or changes in mitochondrial or nuclear position not due to applied membrane stretch. Two consecutive images were acquired one minute apart $(n=3)$ and used for strain calculation independent of applied membrane stretch. For mitochondrial images, a $4 \times 4$ grid of pixels (strain resolution of $2 \mu \mathrm{m}$ ) was selected for displacement tracking (16 measurement points, 10 pixel spacing between grid points chosen over a $36 \mu \mathrm{m}^{2}$ measurement area) lying completely within the cytoplasm of the cell. A total of 12 cells (4 cells from three separate experiments) were analyzed and their strain components compared against zero. For each strain component, the average strain error $\left(E_{\text {actual }}-E_{\text {measured }}\right)$ was computed to determine bias error, and absolute strain error $\left(\left|E_{\text {actual }}-E_{\text {measured }}\right|\right)$ was computed as a measure of precision error arising from sources independent of substrate deformation. The same analysis was repeated for cell nuclei.

Additionally, error analyses were performed to estimate the accuracy of the measurement system following stretch, where image distortion may result in
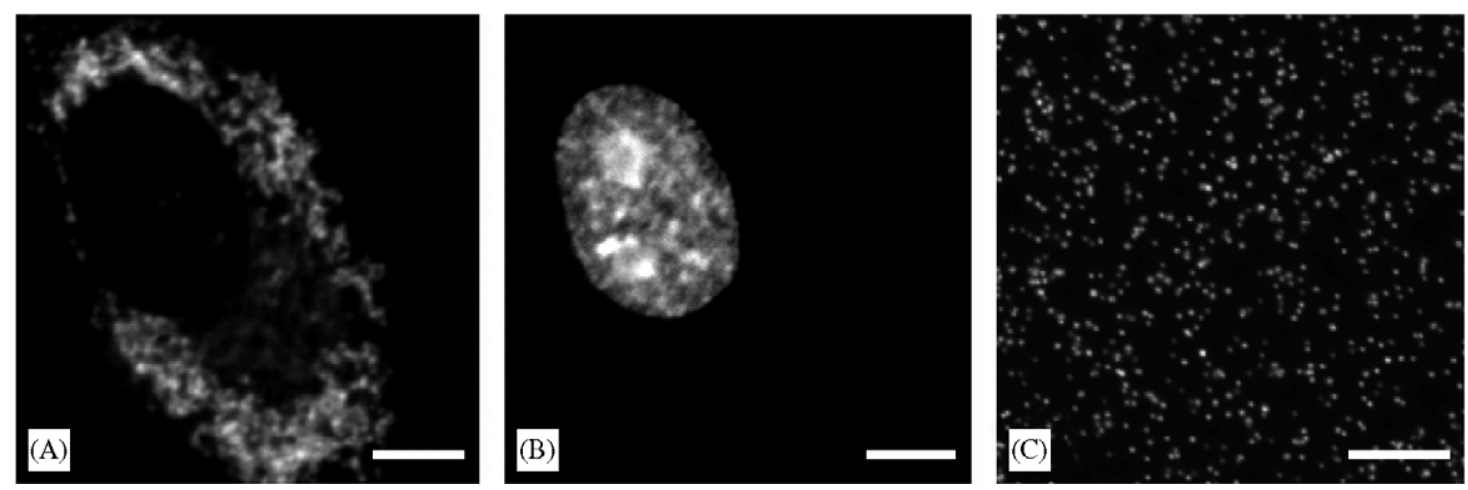

Fig. 2. Representative images of fluorescently labeled (a) mitochondria, (b) nucleus (scale bars $=10 \mu \mathrm{m}$ ), and (c) silicone substrate coated with fluorescent microspheres (scale bar $=100 \mu \mathrm{m}$ ). 
errors in displacement tracking. Consecutive image sets were acquired as described above, and a linear displacement gradient was applied to one of the paired images to simulate a "deformed" image. The linear displacement gradient resulted in a uniform tensile strain in the $x$-direction $\left(E_{x x}\right)$ combined with a uniform compressive strain in the transverse direction $\left(E_{y y}=-E_{x x} / 3\right)$. Three different stretch magnitudes corresponding to image strains, $E_{x x}$ of $0.051,0.105,0.161$ were applied to mitochondrial and nuclear images and strains were calculated as described above $(n=12$ cells). Several subset mask sizes $(m=31,35,37,41$ pixels $)$ were evaluated for each stretch magnitude. Average and absolute strain errors were computed for all components of strain. The subset mask size that resulted in the lowest absolute strain error was selected for use in the experimental analysis.

\subsection{Intracellular strain calculations following stretch}

A total of 56 cells were imaged and analyzed $(n=10$ membranes) on substrates subjected to stretches in the $x$-direction of approximately 1.05 to 1.15 . Strain fields were calculated as described above, tracking a $4 \times 4$ grid of pixels lying entirely within the cell cytoplasm or nucleus. Substrate strain was calculated for each stretch experiment and averaged across an image field matched to the image field of the analyzed cells $(54 \mu \mathrm{m} \times 54 \mu \mathrm{m}$ area, $7 \times 7$ grid, 20 pixel grid spacing). Cytoplasmic and nuclear strains were compared with average substrate strain via linear regression. For an individual cell, the strain transferred from the substrate to the cell was defined as the strain transfer ratio (STR), calculated as the ratio of the mean cell strain (cytoplasmic or nuclear) to the mean underlying substrate strain. STR values were compared to 1 ( $t$-test, $p<0.05$ significant) to test the hypothesis that substrate strain was fully transferred to cell cytoplasm and nucleus. Additionally, pairwise comparisons of STR values were made between cell region (cytoplasm versus nucleus) and direction (parallel and transverse to direction of applied stretch) for each cell (paired $t$-test, Bonferroni correction).

Fibroblast-like cells typically elongate more along one cell axis when attached in monolayer and may exhibit anisotropic mechanical behaviors due to cytoskeletal arrangement about this axis (e.g., actin stress fiber alignment) (Hu et al., 2004). Thus, we tested for correlations of cell alignment with STR for cytoplasm and nuclei. Additionally, cell aspect ratio (cell length/ width) was measured for each cell using SYTO labeled reference images, where length was visually identified as the long axis of the cell, and width was the transverse dimension. The alignment angle $\theta$ for a cell was calculated as the angle between the $x$-direction and the direction of the long axis of the cell, with an angle of $0^{\circ}$ representing alignment with the direction of stretch. The dependence of STR on cell alignment $(\cos (\theta))$ was evaluated via linear and non-linear regression analyses (SigmaPlot, Systat, Pt. Richmond, CA), and by grouping cells based on alignment and comparing via ANOVA. Effects of cell aspect ratio on STR were also examined by repeating the analysis for cells with higher aspect ratios only $(>2.0)$.

\section{Results}

\subsection{Error analyses}

In the absence of applied stretch, the average strain magnitudes in the cytoplasm or nucleus were low $\left(E_{x x}<0.002, E_{y y}<0.0025, E_{x y}<0.001\right)$ and did not differ statistically from zero, indicating no bias error in the system (Table 1). Absolute error in the strain measures was less than 0.007 , indicating low precision error due to sources independent of membrane stretch. Using a simulated strain field, average strain error did not differ from zero for any component of strain, indicating no bias error was introduced with image stretch or strain calculation (Table 1). Absolute strain error increased slightly with increasing magnitude of stretch (error of $\sim 0.006$ for 1.05 axial stretch; error of $\sim 0.009$ for 1.16 axial stretch). Strain errors were similar in magnitude for both cytoplasm and nucleus. Overall, these results provide evidence of an ability to resolve strains of at least 0.01 within cell cytoplasm or nucleus.

\subsection{Cell stretching experiments}

Ten substrate stretch experiments were performed with uniaxial strains that ranged from 0.045 to 0.14 . Substrate strains for the region underlying measured cells (54 $\mu \mathrm{m}$ square) within each experiment were found to be very uniform (e.g., Fig. 3), with standard deviations in $E_{x x}$ for the substrate typically less than $5 \%$ of the mean strain values (e.g., $E_{x x}=0.089 \pm 0.002$, Fig. 4A; $E_{y y}=-0.067 \pm 0.0008$, Fig. 4B).

Strains averaged for the cytoplasmic region within one cell were typically similar to magnitudes for the underlying substrate; however, strain magnitude often varied significantly across the cytoplasmic region. For example, for the four cells shown in Fig. 3, measured cytoplasmic strains ranged from $61 \%$ to $181 \%$ of a cell's mean strain. Overall, standard deviations for cytoplasmic strains (for all cells) were 0.024 for $E_{x x}, 0.014$ for $E_{y y}$ and 0.012 for $E_{x y}$. Furthermore, variations in strain magnitude were also noted across different cells on the same substrate, where mean $E_{x x}$ cytoplasmic strains ranged from $57 \%$ to $112 \%$ of the mean substrate strain (Fig. 3). For $E_{x x}$ in all experiments, cytoplasmic strains were typically equal to or less than those of the substrate (48 out of 56 cells had STR $_{x}$ less than 1.1), although 
Table 1

Average and absolute strain error (mean \pm SD) for measurements within (A) cytoplasm and (B) nucleus for image pairs with simulated applied strain

\begin{tabular}{|c|c|c|c|c|c|c|}
\hline \multirow{2}{*}{$\begin{array}{l}\text { Simulated applied } \\
\text { strain }\left(E_{x x}\right)\end{array}$} & \multicolumn{3}{|c|}{ Average strain error } & \multicolumn{3}{|c|}{ Absolute strain error } \\
\hline & $E_{x x}$ & $E_{y y}$ & $E_{x y}$ & $E_{x x}$ & $E_{y y}$ & $E_{x y}$ \\
\hline \multicolumn{7}{|l|}{ (A) Cytoplasm } \\
\hline 0.000 & $0.0000 \pm 0.0087$ & $0.0020 \pm 0.0078$ & $-0.0008 \pm 0.0052$ & $0.0063 \pm 0.0059$ & $0.0061 \pm 0.0052$ & $0.0042 \pm 0.0031$ \\
\hline 0.051 & $-0.0002 \pm 0.0105$ & $-0.0020 \pm 0.0083$ & $0.0009 \pm 0.0063$ & $0.0076 \pm 0.0072$ & $0.0065 \pm 0.0055$ & $0.0051 \pm 0.0039$ \\
\hline 0.105 & $-0.0009 \pm 0.0113$ & $-0.0031 \pm 0.0067$ & $-0.0003 \pm 0.0069$ & $0.0083 \pm 0.0077$ & $0.0056 \pm 0.0048$ & $0.0055 \pm 0.0041$ \\
\hline 0.161 & $0.0002 \pm 0.0122$ & $-0.0045 \pm 0.0077$ & $0.0009 \pm 0.0068$ & $0.0091 \pm 0.0082$ & $0.0069 \pm 0.0056$ & $0.0054 \pm 0.0042$ \\
\hline \multicolumn{7}{|l|}{ (B) Nucleus } \\
\hline 0.000 & $0.0020 \pm 0.0082$ & $0.0024 \pm 0.0088$ & $-0.0002 \pm 0.0051$ & $0.0055 \pm 0.0063$ & $0.0065 \pm 0.0063$ & $0.0036 \pm 0.0037$ \\
\hline 0.051 & $-0.0020 \pm 0.0090$ & $-0.0028 \pm 0.0079$ & $0.0003 \pm 0.0059$ & $0.0061 \pm 0.0069$ & $0.0058 \pm 0.0061$ & $0.0041 \pm 0.0042$ \\
\hline 0.105 & $-0.0028 \pm 0.0094$ & $-0.0038 \pm 0.0079$ & $0.0003 \pm 0.0054$ & $0.0063 \pm 0.0075$ & $0.0065 \pm 0.0060$ & $0.0038 \pm 0.0039$ \\
\hline 0.161 & $-0.0030 \pm 0.0100$ & $-0.0055 \pm 0.0079$ & $0.0003 \pm 0.0056$ & $0.0067 \pm 0.0079$ & $0.0070 \pm 0.0066$ & $0.0038 \pm 0.0041$ \\
\hline
\end{tabular}

Consecutive images were taken 1 min apart to obtain an image set.

A simulated constant strain field was applied to the second image for determination of measurement errors associated with the imaging system and algorithm $\left(E_{x x}=0.00,0.051,0.105,0.161, E_{y y}=-E_{x x} / 3\right)$.

$n=192$ for each strain level: 12 cells from three separate experiments, 16 strain measurements/cell.
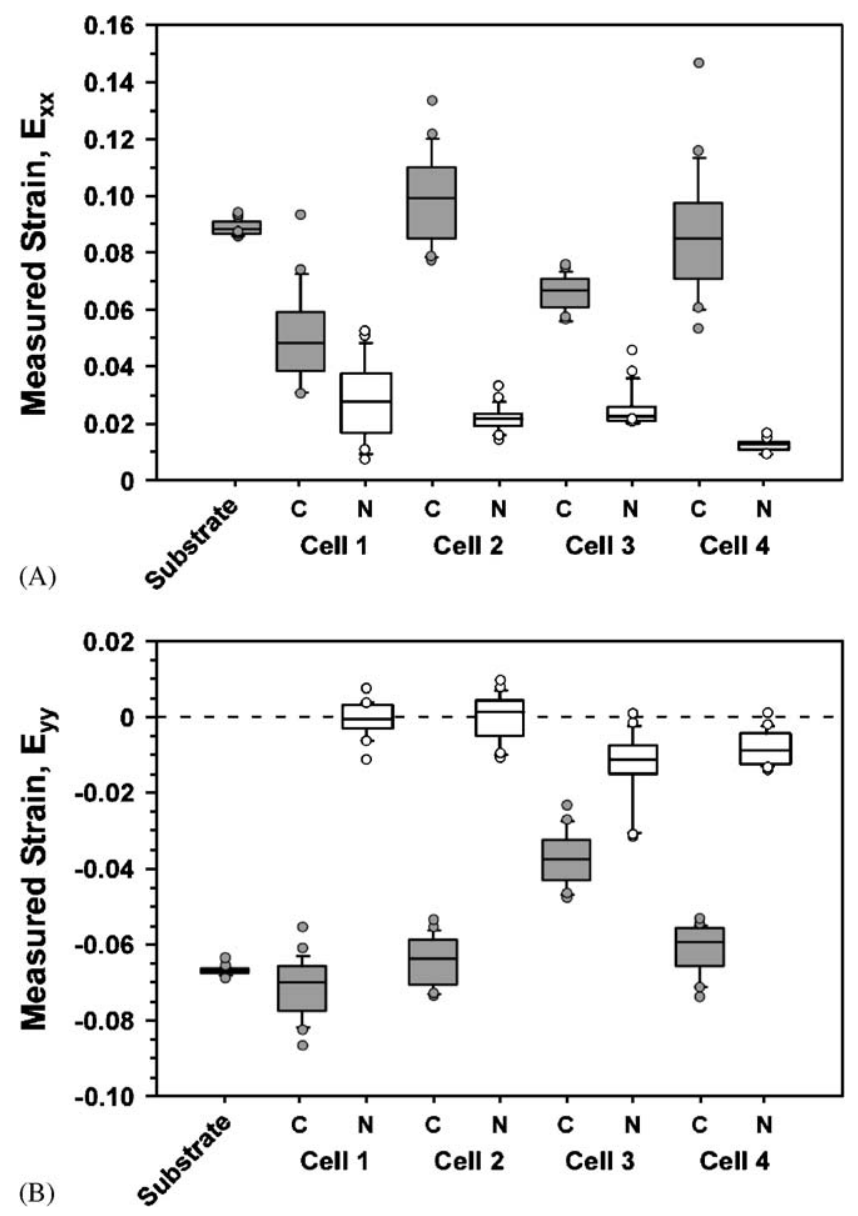

Fig. 3. Measured (A) longitudinal $\left(E_{x x}\right)$ and (B) transverse $\left(E_{y y}\right)$ substrate and cell strains for a representative uniaxial stretch experiment. For each cell, measured cytoplasmic (C, shaded box) and nuclear ( $\mathrm{N}$, unshaded box) strain measurements are shown. Box plot shows inter-quartile range (box) and median (line). Whiskers identify 90th percentile range, with data points outside of this range shown (o). $n=16$ strain measurements per cell for cytoplasm and nucleus; $n=49$ strain measurements per substrate.

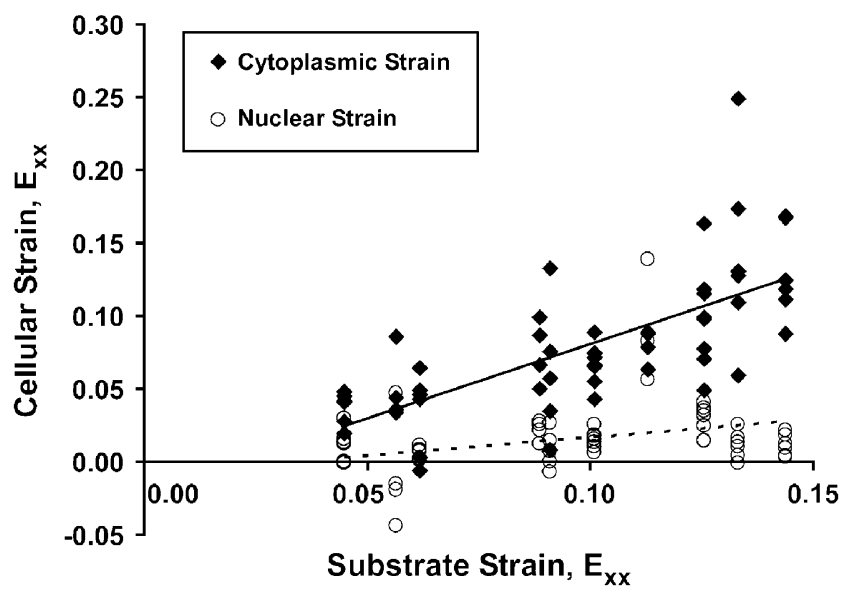

Fig. 4. Measured mean substrate strain versus measured mean cytoplasmic and nuclear strains for cells subjected to uniaxial stretch (in $x$-direction). Data shown represents ten separate stretch experiments and a total of 56 cells. Measured cytoplasmic strains $(\diamond)$ were found to correspond with substrate strain (solid line, slope $=1.02$, $\left.p<0.0001, R^{2}=0.36\right)$. Measured nuclear strains $(O)$ for same cells were much lower but did correspond with substrate strain (dashed line, slope $\left.=0.25, p<0.0001, R^{2}=0.08\right)$.

several cells exhibited extreme behaviors (one cell with $\mathrm{STR}_{x}$ as high as 1.88 , and another less than 0 ). The mean STR for cytoplasmic strain ( $x$-direction) was found to be $\mathrm{STR}_{x}=0.79 \pm 0.34$, which was significantly less than $1(p<0.0001)$, indicating cytoplasmic strains in the direction of applied stretch were not fully transferred. Transversely ( $y$-direction), $\mathrm{STR}_{y}=0.99 \pm 0.68$ was not different from $1 \quad(p=0.88)$. A significant difference was not detected between $\mathrm{STR}_{x}$ and $\mathrm{STR}_{y}$ for the cytoplasm $(p>0.05)$.

Nuclear strains were found to be much lower than that of the substrate or cytoplasm, with $\mathrm{STR}_{x}=$ $0.17 \pm 0.28$ and $\mathrm{STR}_{y}=0.38 \pm 0.89$. These values were 
significantly different from 1 and $0(p<0.0001)$, lower than those of the cytoplasm $\left(p<0.0001\right.$ for STR $_{x}$ and $\mathrm{STR}_{y}$ ), with no difference detected between $\mathrm{STR}_{x}$ and $\operatorname{STR}_{y}(p>0.05)$. Additionally, nuclear strains were more uniform within a given cell, with standard deviations for nuclear strains of 0.010 for $E_{x x}, 0.011$ for $E_{y y}$ and 0.007 for $E_{x y}$.

Cytoplasmic strains in the $x$-direction were significantly correlated with substrate strains using data for all cells from all experiments (slope $=1.02, p<0.0001$, $R^{2}=0.36 ; n=896$ each for cytoplasm and nucleus) (Fig. 4), with a slope that was not significantly different from $1(p=0.68)$. The $x$-intercept of the best linear-fit was found to the right of the origin $(x=0.021$, $p<0.0001$ ), suggesting that a nonlinear relationship between cell strain and substrate strain may exist at low strain levels. Nuclear strain ( $x$-direction) was weakly correlated with substrate strains (slope $=0.25$, $\left.p<0.0001, R^{2}=0.08\right)$, with an $x$-intercept of 0.032 that was significantly different from zero $(p<0.01)$.

There was no evidence of a relationship between cytoplasmic or nuclear $\mathrm{STR}_{x}$ and cell alignment (Fig. $\left.5 \mathrm{~A}, p=0.14, R^{2}=0.041\right)$; however, analysis of variance was able to detect differences in cytoplasmic $\mathrm{STR}_{x}$ amongst two groups of approximately equal cell numbers ("aligned" group: $-45<\theta<+45, \quad n=24$; "unaligned" group: $\theta<-45, \quad \theta>+45, \quad n=31$, $p=0.015$, Fig. 5B). One cell had no polarity and was excluded from this analysis. Thus, an effect of cell alignment on cytoplasmic strain transfer was detected, with higher strain transfers for the group of "aligned" cells. In the direction transverse to applied stretch $\left(\mathrm{STR}_{y}\right)$, "unaligned" cells showed a trend towards higher $\mathrm{STR}_{y}$ as compared to "aligned cells", although these differences were not detected as significant (Fig. 5B). Cell aspect ratios were highly variable, with a mean of $2.37 \pm 0.96$. Regression analyses between cell alignment and STR were not changed by considering only cells with high aspect ratios.

\section{Discussion}

This study describes a novel method to quantify strain fields in cells at the subcellular level using fluorescent cell labeling and texture correlation analyses. Strain was calculated at both cytoplasmic and nuclear regions within cells following uniaxial substrate stretch, and compared to values for substrate strain. Cytoplasmic strains in the direction of applied uniaxial stretch were found to be somewhat lower than the underlying substrate $\left(\mathrm{STR}_{x}=0.79\right)$, indicating substrate strains

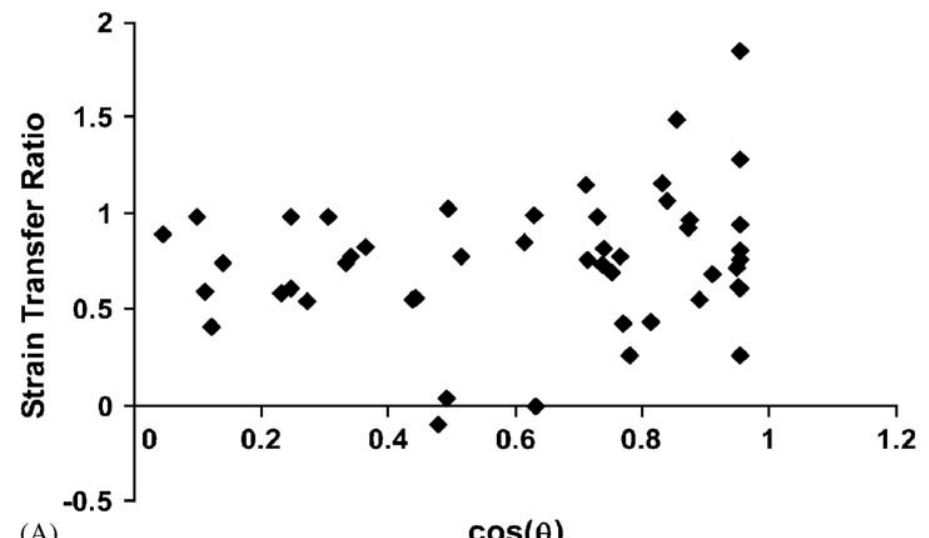

(A)

$\cos (\theta)$

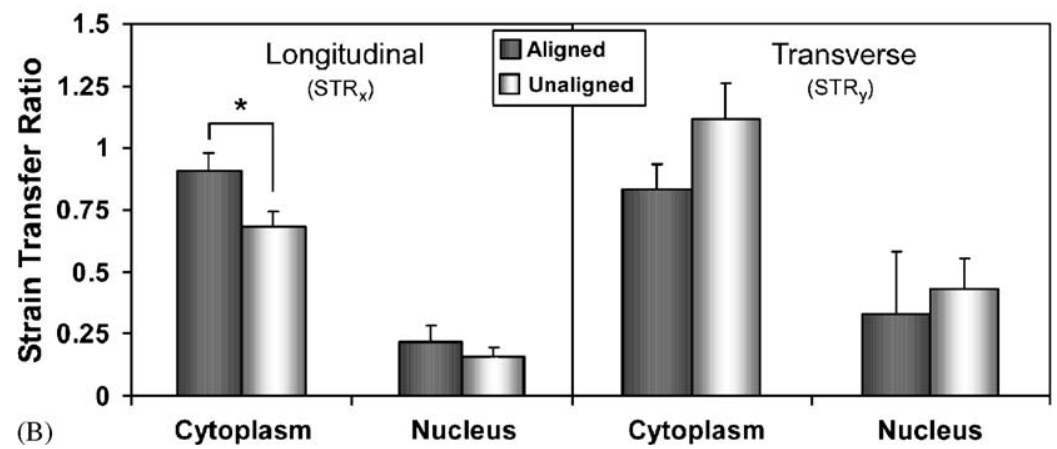

Fig. 5. Effects of cell alignment on strain transfer from substrate for cells subjected to uniaxial stretch. (A) Strain transfer ratio in direction of applied stretch ( $x$-direction) for cell cytoplasm, $n=55$ cells. (B) Mean cytoplasmic and nuclear strain transfer ratios $( \pm$ SEM) longitudinal ( $x$-direction) and transverse ( $y$-direction) to direction of applied substrate stretch for "aligned" $(-45<\theta<+45, n=24)$ and "unaligned" groups, $(n=31, \theta<-45$, $\theta>+45),{ }^{*} p=0.015$, ANOVA. 
were not fully transferred to the cytoplasm. However, regression analyses (Fig. 4) revealed that strain was transferred from substrate to cytoplasm at approximately a $1: 1$ ratio $($ slope $=1.02$ ), after some offset or nonlinear behavior at low strain levels. Further study of strain transfer at strain levels less than 0.04 may be necessary to fully characterize this behavior. In contrast to cytoplasmic strain, nuclear strains were always small and weakly correlated to substrate deformation (slope $=0.25$ ), with a mean STR of 0.17 .

Cells with a higher orientation along the long axis of stretch were associated with higher STR for the cytoplasmic regions, than for non-aligned cells. This finding implies that coupling between the cell and substrate will be stronger for these cases of more highly aligned cells. The extent of cell alignment, as well as value for cell aspect ratio, may indirectly imply underlying mechanical anisotropy along the cell's long axis (Hu et al., 2004). Direct measurement of cell components affecting cell mechanical anisotropy, such as actin stress fiber alignment, stress fiber number or focal adhesion densities may be valuable parameters in elucidating these potential effects of cell anisotropy on strain transfer. Cells expressing GFP-labeled cytoskeletal or adhesion proteins may be of great use for identifying the effects of structural heterogeneity on local strain transfer behavior.

Determination of error in strain measurement for the technique presented here was challenging due to the lack of a high-resolution "gold standard" with which to compare. The error analysis undertaken for this study used consecutive images acquired over time (with no applied substrate stretch) to account for errors due to independent mitochondrial movement and optical imaging, and used artificially "stretched" images to detect compounding effects of image distortion. Our error analyses suggest the strain measurement technique presented in this study is both accurate and precise for measuring intracellular strains in the range of $0.02-0.20$, with overall bias and precision errors less than 0.005 and 0.01 , respectively (Table 1). These results suggest the present technique is sufficiently precise to resolve intracellular strains of greater than 0.01 within subcellular regions as small as $4 \mu \mathrm{m}^{2}$. Additionally, increases in measurement resolution may be possible by increasing image resolution via a higher-powered microscope objective.

Mean cytoplasmic strains often varied substantially among cells on a given substrate, and among cytoplasmic regions within a single cell. These strain differences contributed to large variability in strain measurements that were much larger than our estimates of error due to measurement technique. Differences in cytoplasmic regions within individual cells may reflect biological variability of cytoskeletal filament arrangement or organelle positioning, as the resolution of the current measurement technique may be high enough to detect localized differences in mechanical deformation at sites of local highly organized structures (e.g., actin stress fibers, vacuoles). In particular, the mitochondria used to track displacements in this study are typically bound to microtubules (Bereiterhahn and Voth, 1994), and localized mitochondrial displacement could be a result of microtubule buckling (Maniotis et al., 1997; Chen and Ingber, 1999). Additionally, active cytoskeletal reorganization in response to stretch could also contribute to both intracellular and substrate-cell variabilities, and may be the most likely explanation for cells exhibiting extreme mean STR values.

A primary finding of this study is that nuclear strains were small and relatively uniform within a given cell, as compared to the surrounding cytoplasm. These results confirm previous findings (e.g. Maniotis et al., 1997; Caille et al., 1998; Hu et al., 2005) that a linkage exists between the extracellular substrate and the cell nuclei to transfer mechanical deformations. However, only a small amount $(<25 \%)$ of the cytoplasmic strain was transferred to the nucleus. These results correspond with a previous study (Caille et al., 1998) where endothelial cell nuclei were found to deform significantly less (50$80 \%$ less) than the underlying flexible substrate. Similarly, compression of articular cartilage showed less deformation of the chondrocyte nuclei as compared to that of the whole cell (Guilak, 1995). Smaller nuclear strains may be due to significantly higher mechanical stiffness of the nucleus, as reported for a variety of cell types and testing configurations (Dong et al., 1991; Guilak et al., 2000; Caille et al., 2002). Alternatively, the smaller magnitudes of nuclear strain may indicate limited or indirect cytoskeletal connection between cytoplasm and nucleus. Nuclear strain distributions were significantly more uniform than the highly variable strains in the surrounding cytoplasm, with standard deviations of nuclear strain less than $50 \%$ of those in the cytoplasm. This finding may also indicate nuclear mechanical homogeneity at the scale of the strain measurements and suggests mechanical deformations may not be localized to particular subnuclear regions.

In this study, the 2D components of finite strain were calculated, which do not account for out-of-plane displacements that may occur during substrate stretch (e.g., cell flattening). As cells were typically in a fully spread conformation (3-4 $\mu \mathrm{m}$ thickness), errors due to out-of-plane displacements were likely small. The technique described here may be readily adapted to study alternate loading configurations (e.g., biaxial stretch), substrate ligands or transient events, which will be useful for determining mechanisms by which cells transduce specific mechanical stimuli.

The results of the present study should be interpreted with respect to cell phenotype, as other cell types with differing morphologies and cytoskeletal architecture 
(e.g. articular chondrocytes, nucleus pulposus cells) may exhibit different strain transfer behaviors. Additionally, it is currently unclear whether $3 \mathrm{D}$ culture conditions, which more closely represent the in vivo environment of many cell types, would transmit similar levels and patterns of strain to the cell nucleus or cytoplasm (Guilak and Mow, 2000). In these situations, factors such as ligand densities, differing cell adhesions (Cukierman et al., 2001), reduced substrate stiffness or altered cytoskeletal structure may influence intracellular strains as compared to $2 \mathrm{D}$ cultures on rigid substrates. The technique presented here may be extended to $3 \mathrm{D}$ to allow for future investigations of ECM-cell strain transfer in these conformations using 3D matrices or intact tissues, providing further insight into cellular deformation states and mechanotransduction processes.

\section{Acknowledgments}

This work was supported by NIH Grants AR47442, AG15768, AR48182, AR50245 and T32-GM08555.

\section{References}

Baer, A.E., Wang, J.Y., Kraus, V.B., Setton, L.A., 2001. Collagen gene expression and mechanical properties of intervertebral disc cellalginate cultures. Journal of Orthopaedic Research 19 (1), 2-10.

Banes, A.J., Gilbert, J., Taylor, D., Monbureau, O., 1985. A new vacuum-operated stress-providing instrument that applies static or variable duration cyclic tension or compression to cells in vitro. Journal of Cell Science 75, 35-42.

Bay, B.K., 1995. Texture correlation: a method for the measurement of detailed strain distributions within trabecular bone. Journal of Orthopaedic Research 13 (2), 258-267.

Bereiterhahn, J., Voth, M., 1994. Dynamics of mitochondria in living cells - shape changes, dislocations, fusion, and fission of mitochondria. Microscopy Research and Technique 27 (3), 198-219.

Bershadsky, A.D., Balaban, N.Q., Geiger, B., 2003. Adhesiondependent cell mechanosensitivity. Annual Review of Cell and Developmental Biology 19, 677-695.

Bey, M.J., Song, H.K., Wehrli, F.W., Soslowsky, L.J., 2002. A noncontact, nondestructive method for quantifying intratissue deformations and strains. Journal of Biomechanical Engineering 124 (2), 253-258.

Bottlang, M., Simnacher, M., Schmitt, H., Brand, R.A., Claes, L., 1997. A cell strain system for small homogeneous strain applications. Biomedizinische Technik (Berl) 42 (11), 305-309.

Brown, T.D., 2000. Techniques for mechanical stimulation of cells in vitro: a review. Journal of Biomechanics 33 (1), 3-14.

Brown, T.D., Bottlang, M., Pedersen, D.R., Banes, A.J., 2000. Development and experimental validation of a fluid/structureinteraction finite element model of a vacuum-driven cell culture mechanostimulus system. Computer Methods in Biomechanics and Biomedical Engineering 3 (1), 65-78.

Caille, N., Tardy, Y., Meister, J.J., 1998. Assessment of strain field in endothelial cells subjected to uniaxial deformation of their substrate. Annals of Biomedical Engineering 26 (3), 409-416.

Caille, N., Thoumine, O., Tardy, Y., Meister, J.J., 2002. Contribution of the nucleus to the mechanical properties of endothelial cells. Journal of Biomechanics 35 (2), 177-187.
Chen, C.S., Ingber, D.E., 1999. Tensegrity and mechanoregulation: from skeleton to cytoskeleton. Osteoarthritis Cartilage 7 (1), 81-94.

Cukierman, E., Pankov, R., Stevens, D.R., Yamada, K.M., 2001. Taking cell-matrix adhesions to the third dimension. Science 294 (5547), 1708-1712.

Dong, C., Skalak, R., Sung, K.L., 1991. Cytoplasmic rheology of passive neutrophils. Biorheology 28 (6), 557-567.

Estes, B.T., Gimble, J.M., Guilak, F., 2004. Mechanical signals as regulators of stem cell fate. Current Topics in Developmental Biology 60, 91-126.

Gilbert, J.A., Weinhold, P.S., Banes, A.J., Link, G.W., Jones, G.L., 1994. Strain profiles for circular cell culture plates containing flexible surfaces employed to mechanically deform cells in vitro. Journal of Biomechanics 27 (9), 1169-1177.

Gilchrist, C.L., Xia, J.Q., Setton, L.A., Hsu, E.W., 2004. Highresolution determination of soft tissue deformations using MRI and first-order texture correlation. IEEE Transactions on Medical Imaging 23 (5), 546-553.

Guilak, F., 1995. Compression-induced changes in the shape and volume of the chondrocyte nucleus. Journal of Biomechanics 28 (12), 1529.

Guilak, F., Mow, V.C., 2000. The mechanical environment of the chondrocyte: a biphasic finite element model of cell-matrix interactions in articular cartilage. Journal of Biomechanics 33 (12), 1663-1673.

Guilak, F., Ratcliffe, A., Mow, V.C., 1995. Chondrocyte deformation and local tissue strain in articular cartilage: a confocal microscopy study. Journal of Orthopaedic Research 13 (3), 410-421.

Guilak, F., Sah, R.L., Setton, L.A., 1997. Physical regulation of cartilage metabolism. In: Mow, V.C., Hayes, W.C. (Eds.), Basic Orthopaedic Biomechanics. Lippincott-Raven, Philadelphia, pp. 179-207.

Guilak, F., Tedrow, J.R., Burgkart, R., 2000. Viscoelastic properties of the cell nucleus. Biochemical and Biophysical Research Communications 269 (3), 781-786.

Helmke, B.P., Rosen, A.B., Davies, P.F., 2003. Mapping mechanical strain of an endogenous cytoskeletal network in living endothelial cells. Biophysical Journal 84 (4), 2691-2699.

Horner, H.A., Roberts, S., Bielby, R.C., Menage, J., Evans, H., Urban, J.P., 2002. Cells from different regions of the intervertebral disc: effect of culture system on matrix expression and cell phenotype. Spine 27 (10), 1018-1028.

Hsieh, M.H., Nguyen, H.T., 2005. Molecular mechanism of apoptosis induced by mechanical forces. International Review of Cytology 245, 45-90.

Hu, S., Chen, J., Butler, J.P., Wang, N., 2005. Prestress mediates force propagation into the nucleus. Biochemical and Biophysical Research Communications 329 (2), 423-428.

Hu, S.H., Chen, J.X., Fabry, B., Numaguchi, Y., Gouldstone, A., Ingber, D.E., Fredberg, J.J., Butler, J.P., Wang, N., 2003. Intracellular stress tomography reveals stress focusing and structural anisotropy in cytoskeleton of living cells. American Journal of Physiology — Cell Physiology 285 (5), C1082-C1090.

Hu, S.H., Eberhard, L., Chen, J.X., Love, J.C., Butler, J.P., Fredberg, J.J., Whitesides, G.M., Wang, N., 2004. Mechanical anisotropy of adherent cells probed by a three-dimensional magnetic twisting device. American Journal of Physiology _ Cell Physiology 287 (5), C1184-C1191.

Hung, C.T., Williams, J.L., 1994. A method for inducing equi-biaxial and uniform strains in elastomeric membranes used as cell substrates. Journal of Biomechanics 27 (2), 227-232.

Iqbal, J., Zaidi, M., 2005. Molecular regulation of mechanotransduction. Biochemical and Biophysical Research Communications 328 (3), 751-755.

Jahangir, A., Lee, J.M., Waldman, S.D., Anderson, G.I., 2002. Mechanical characterization of a novel cell stimulating system (CSS) to apply dynamic, uniform and isotropic biaxial strains to cells in vitro. Biomedical Sciences Instrumentation 38, 215-220. 
Katsumi, A., Orr, A.W., Tzima, E., Schwartz, M.A., 2004. Integrins in mechanotransduction. Journal of Biological Chemistry 279 (13), 12001-12004.

Lee, A.A., Delhaas, T., Waldman, L.K., MacKenna, D.A., Villarreal, F.J., McCulloch, A.D., 1996. An equibiaxial strain system for cultured cells. American Journal of Physiology-Cell Physiology 271 (4 Pt 1), C1400-C1408.

Li, Y.S., Haga, J.H., Chien, S., 2005. Molecular basis of the effects of shear stress on vascular endothelial cells. Journal of Biomechanics 38 (10), 1949-1971.

Maniotis, A.J., Chen, C.S., Ingber, D.E., 1997. Demonstration of mechanical connections between integrins cytoskeletal filaments, and nucleoplasm that stabilize nuclear structure. Proceedings of the National Academy of Sciences 94 (3), 849-854.

Sachs, F., Morris, C.E., 1998. Mechanosensitive ion channels in nonspecialized cells. Reviews of Physiology, Biochemistry and Pharmacology 132, 1-77.
Shemesh, T., Geiger, B., Bershadsky, A.D., Kozlov, M.M., 2005. Focal adhesions as mechanosensors: a physical mechanism. Proceedings of the National Academy of Sciences.

Vande Geest, J.P., Di Martino, E.S., Vorp, D.A., 2004. An analysis of the complete strain field within Flexercell membranes. Journal of Biomechanics 37 (12), 1923-1928.

Wang, N., Suo, Z., 2005. Long-distance propagation of forces in a cell. Biochemical and Biophysical Research Communications 328 (4), 1133-1138.

Wang, J.Y., Baer, A.E., Kraus, V.B., Setton, L.A., 2001. Intervertebral disc cells exhibit differences in gene expression in alginate and monolayer culture. Spine 26 (16), 1747-1751 (discussion 1752).

Wang, C.C., Chahine, N.O., Hung, C.T., Ateshian, G.A., 2003. Optical determination of anisotropic material properties of bovine articular cartilage in compression. Journal of Biomechanics 36 (3), 339-353. 